\title{
Positive answer for a conjecture about stabilizable means
}

\section{Mustapha Raïssouli*}

\begin{abstract}
In an earlier paper (Raïssouli in Appl. Math. E-Notes 11:159-174, 2011), the author conjectured that for given stable means $m_{1}$ and $m_{2}$ such that $m_{1} \leq m_{2}$, there exists a unique $\left(m_{1}, m_{2}\right)$-stabilizable mean satisfying that $m_{1} \leq m \leq m_{2}$. In the present paper, a positive answer of this conjecture is given. Some examples, illustrating the theoretical study, are discussed.
\end{abstract}

MSC: $26 \mathrm{E} 60$

Keywords: means; stable means; stabilizable means

\section{Introduction}

In the recent past, the theory of means has been the subject of intensive research. Stability and stabilizability concepts for means have been recently introduced by the author in [1]. It has been proved to be a useful tool for theoretical viewpoint as well as for practical purposes [2-4]. In this section, we recall some basic notions about means in two variables that will be needed later. We understand by (bivariate) mean a binary map $m$ between positive real numbers satisfying the following statements:

(i) $m(a, a)=a$ for all $a>0$;

(ii) $m(a, b)=m(b, a)$ for all $a, b>0$;

(iii) $m(t a, t b)=t m(a, b)$ for all $a, b, t>0$;

(iv) $m(a, b)$ is an increasing function in $a$ (and in $b$ );

(v) $m(a, b)$ is a continuous function of $a$ and $b$.

The maps $(a, b) \longmapsto \min (a, b)$ and $(a, b) \longmapsto \max (a, b)$ are (trivial) means which are denoted by min and max, respectively. The standard examples of means are given in the following, see [5-24] for instance.

$$
\begin{aligned}
& A:=A(a, b)=\frac{a+b}{2} ; \quad G:=G(a, b)=\sqrt{a b} ; \quad H:=H(a, b)=\frac{2 a b}{a+b} ; \\
& L:=L(a, b)=\frac{b-a}{\ln b-\ln a}, \quad L(a, a)=a ; \quad I:=I(a, b)=\frac{1}{e}\left(\frac{b^{b}}{a^{a}}\right)^{1 /(b-a)}, \quad I(a, a)=a ;
\end{aligned}
$$

and are known as the arithmetic, geometric, harmonic, logarithmic and identric means, respectively.

For two means $m_{1}$ and $m_{2}$, we write $m_{1} \leq m_{2}$ if and only if $m_{1}(a, b) \leq m_{2}(a, b)$ for every $a, b>0$ and $m_{1}<m_{2}$ if and only if $m_{1}(a, b)<m_{2}(a, b)$ for all $a, b>0$ with $a \neq b$. The above 
means satisfy the known chain of inequalities

$\min <H<G<L<I<A<\max$.

We say that $m$ is a strict mean if $m(a, b)$ is strictly increasing in $a$ and in $b$. Also, every strict mean $m$ satisfies that $m(a, b)=a \Longrightarrow a=b$. It is not hard to check that the trivial means min and max are not strict, while $A, G, H, L, I, S, C$ are strict means.

For the sake of simplicity for the reader, we end this section by recalling some basic notions and results stated by the author in an earlier paper [1] and needed in the sequel.

Definition 1.1 Let $m_{1}, m_{2}$ and $m_{3}$ be three given means. For all $a, b>0$, define

$$
\mathcal{R}\left(m_{1}, m_{2}, m_{3}\right)(a, b)=m_{1}\left(m_{2}\left(a, m_{3}(a, b)\right), m_{2}\left(m_{3}(a, b), b\right)\right)
$$

called the resultant mean-map of $m_{1}, m_{2}$ and $m_{3}$.

A study investigating the elementary properties of the resultant mean-map was stated in [1]. Here, we just recall the following result needed later.

Proposition 1.1 Let $m_{1}, m_{2}$ and $m_{3}$ be three means. Then the map $(a, b) \longmapsto \mathcal{R}\left(m_{1}, m_{2}\right.$, $\left.m_{3}\right)(a, b)$ defines a mean. Further the mean-map $\left(m_{1}, m_{2}, m_{3}\right) \longmapsto \mathcal{R}\left(m_{1}, m_{2}, m_{3}\right)$ is pointwisely increasing with respect to each of its mean variables, that is,

$$
\left(m_{1} \leq m_{1}^{\prime}, m_{2} \leq m_{2}^{\prime}, m_{3} \leq m_{3}^{\prime}\right) \quad \Longrightarrow \quad \mathcal{R}\left(m_{1}, m_{2}, m_{3}\right) \leq \mathcal{R}\left(m_{1}^{\prime}, m_{2}^{\prime}, m_{3}^{\prime}\right)
$$

In $[1,4]$, the author gives a lot of examples about computations of $\mathcal{R}\left(m_{1}, m_{2}, m_{3}\right)$ when $m_{1}, m_{2}$ and $m_{3}$ are means belonging to the set of the above standard means.

As proved in $[1,3,4]$, and will be again shown throughout this paper, the resultant meanmap stems its importance in the fact that it is a good tool for introducing the stability and stabilizability concepts as recalled below.

Definition 1.2 A mean $m$ is said to be:

(a) stable if $\mathcal{R}(m, m, m)=m$;

(b) stabilizable if there exist two nontrivial stable means $m_{1}$ and $m_{2}$ satisfying the relation $\mathcal{R}\left(m_{1}, m, m_{2}\right)=m$. We then say that $m$ is $\left(m_{1}, m_{2}\right)$-stabilizable.

In $[1,3]$, the author stated a developed study about the stability and stabilizability of the above standard means. In particular, he proved the following.

Theorem 1.2 With the above, the following assertions hold true:

(1) The arithmetic, geometric and harmonic means $A, G$ and $H$ are stable.

(2) The logarithmic mean $L$ is $(H, A)$-stabilizable and $(A, G)$-stabilizable.

(3) The identric mean I is $(G, A)$-stabilizable.

\section{Two needed results}

The next definition [2], recalling another concept for means, will be needed in the sequel. 
Definition 2.1 Let $m_{1}$ and $m_{2}$ be two means. The tensor product of $m_{1}$ and $m_{2}$ is the map, denoted $m_{1} \otimes m_{2}$, defined by

$$
\forall a, b, c, d>0 \quad m_{1} \otimes m_{2}(a, b, c, d)=m_{1}\left(m_{2}(a, b), m_{2}(c, d)\right) .
$$

A mean $m$ will be called cross mean if the map $m^{\otimes 2}:=m \otimes m$ is symmetric with its four variables.

It is proved in [1] that every cross mean is stable. The reverse of this latter assertion is still an open problem.

Now, let $m_{1}, m_{2}$ and $m_{3}$ be three given means. For the sake of simplicity, we set

$$
\mathcal{M}\left(m_{1}, m_{2}, m_{3}\right):=m_{1}\left(m_{2}, m_{3}\right)
$$

in the sense that

$$
\mathcal{M}\left(m_{1}, m_{2}, m_{3}\right)(a, b):=m_{1}\left(m_{2}(a, b), m_{3}(a, b)\right)
$$

for all $a, b>0$.

Clearly, $\mathcal{M}\left(m_{1}, m_{2}, m_{2}\right)=m_{2}$ for all means $m_{1}$ and $m_{2}$. If $m_{1}$ is a strict mean, then $\mathcal{M}\left(m_{1}, m_{2}, m_{3}\right)=m_{2}$ (resp. $\left.\mathcal{M}\left(m_{1}, m_{2}, m_{3}\right)=m_{3}\right)$ if and only if $m_{2}=m_{3}$. Further, we have $\mathcal{M}\left(m_{1}, m_{2}, m_{3}\right)=\mathcal{M}\left(m_{1}, m_{3}, m_{2}\right)$ for all means $m_{2}$ and $m_{3}$.

The next result will be of interest later.

Proposition 2.1 Let $m_{1}$ and $m_{2}$ be two given means. Assume that $m_{1}$ is a cross mean, then we have

$$
\mathcal{R}\left(m_{1}, m_{1}, m_{2}\right)=\mathcal{M}\left(m_{1}, m_{1}, m_{2}\right) .
$$

Proof By Definition 1.1 and Definition 1.2, one has, for all $a, b>0$,

$$
\begin{aligned}
\mathcal{R}\left(m_{1}, m_{1}, m_{2}\right)(a, b) & =m_{1}\left(m_{1}\left(a, m_{2}(a, b)\right), m_{1}\left(m_{2}(a, b), b\right)\right) \\
& =m_{1} \otimes m_{1}\left(a, m_{2}(a, b), m_{2}(a, b), b\right) .
\end{aligned}
$$

Since $m_{1}$ is a cross mean, then

$$
\begin{aligned}
\mathcal{R}\left(m_{1}, m_{1}, m_{2}\right)(a, b) & =m_{1} \otimes m_{1}\left(a, b, m_{2}(a, b), m_{2}(a, b)\right) \\
& =m_{1}\left(m_{1}(a, b), m_{2}(a, b)\right)=\mathcal{M}\left(m_{1}, m_{1}, m_{2}\right)(a, b),
\end{aligned}
$$

which concludes the proof.

The above proposition implies again that every cross mean is stable. The next theorem is more interesting.

Theorem 2.2 Let $m_{1}, m_{2}, m_{3}$ and $m_{4}$ be four given means. Assume that $m_{1}$ is a cross mean, then the following holds:

$$
\mathcal{R}\left(m_{1}, \mathcal{M}\left(m_{1}, m_{3}, m_{4}\right), m_{2}\right)=\mathcal{M}\left(m_{1}, \mathcal{R}\left(m_{1}, m_{3}, m_{2}\right), \mathcal{R}\left(m_{1}, m_{4}, m_{2}\right)\right) .
$$


Proof By Definition 1.1, we have, for all $a, b>0$,

$$
\begin{aligned}
\mathcal{R} & \left(m_{1}, \mathcal{M}\left(m_{1}, m_{3}, m_{4}\right), m_{2}\right)(a, b) \\
& =m_{1}\left(\mathcal{M}\left(m_{1}, m_{3}, m_{4}\right)\left(a, m_{2}(a, b)\right), \mathcal{M}\left(m_{1}, m_{3}, m_{4}\right)\left(m_{2}(a, b), b\right)\right),
\end{aligned}
$$

which with (2.1) gives

$$
\begin{aligned}
\mathcal{R} & \left(m_{1}, \mathcal{M}\left(m_{1}, m_{3}, m_{4}\right), m_{2}\right)(a, b) \\
& =m_{1}\left(m_{1}\left(m_{3}\left(a, m_{2}(a, b)\right), m_{4}\left(a, m_{2}(a, b)\right)\right), m_{1}\left(m_{3}\left(m_{2}(a, b), b\right), m_{4}\left(m_{2}(a, b), b\right)\right)\right) .
\end{aligned}
$$

Since $m_{1}$ is a cross mean, then we have

$$
\begin{aligned}
\mathcal{R} & \left(m_{1}, \mathcal{M}\left(m_{1}, m_{3}, m_{4}\right), m_{2}\right)(a, b) \\
& =m_{1}\left(m_{1}\left(m_{3}\left(a, m_{2}(a, b)\right), m_{3}\left(m_{2}(a, b), b\right)\right), m_{1}\left(m_{4}\left(a, m_{2}(a, b)\right), m_{4}\left(m_{2}(a, b), b\right)\right)\right) .
\end{aligned}
$$

Again, by Definition 1.1 and (2.1), respectively, we obtain

$$
\begin{aligned}
\mathcal{R}\left(m_{1}, \mathcal{M}\left(m_{1}, m_{3}, m_{4}\right), m_{2}\right)(a, b) & =m_{1}\left(\mathcal{R}\left(m_{1}, m_{3}, m_{2}\right)(a, b), \mathcal{R}\left(m_{1}, m_{4}, m_{2}\right)(a, b)\right) \\
& =\mathcal{M}\left(m_{1}, \mathcal{R}\left(m_{1}, m_{3}, m_{2}\right), \mathcal{R}\left(m_{1}, m_{4}, m_{2}\right)\right)(a, b),
\end{aligned}
$$

which completes the proof.

In [1], for defining an $\left(m_{1}, m_{2}\right)$-stabilizable mean, the author imposed that the means $m_{1}$ and $m_{2}$ should be nontrivial and stable. The fact that $m_{1}$ and $m_{2}$ are nontrivial is clear since the relation $\mathcal{R}(\min , m, \max )=m$ is valid for every mean $m$. However, the fact that $m_{1}$ and $m_{2}$ are stable was imposed only in the aim to characterize a stabilizable mean $m$ (as $L$ and $I$ ) in terms of $m_{1}$ and $m_{2}$ having simple expressions (as $A, G$ and $H$ ). As example, we know that $L$ is $(H, A)$-stabilizable, where $H$ and $A$ are (stable) means having expressions more simple as that of $L$. Analogous way for the fact that $L$ is $(A, G)$-stabilizable and $I$ is $(G, A)$-stabilizable can be stated.

\section{Existence and uniqueness of a stabilizable mean}

In [1], the author stated the following conjecture.

Conjecture Let $m_{1}$ and $m_{2}$ be two nontrivial stable means such that $m_{1} \leq m_{2}$. Then there exists one and only one mean $m$, which is $\left(m_{1}, m_{2}\right)$-stabilizable, satisfying that $m_{1} \leq$ $m \leq m_{2}$.

The aim of this section is to prove that the above conjecture is true when we add convenient hypotheses for the means $m_{1}$ and $m_{2}$. Of course, following Definition $1.2, m_{1}$ and $m_{2}$ will be assumed to be stable means. We can ask why it is interesting to solve the above conjecture. In fact, as we have seen before, the means $L$ and $I$, having complicated expressions, are stabilizable with respect to $A, G, H$ whose expressions are more simple. It follows that if for given (simple) means $m_{1}$ and $m_{2}$ we show that there exists a unique 
$\left(m_{1}, m_{2}\right)$-stabilizable mean, we can then characterize new means in terms of known (simple) means. This can be also useful when we speak for means involving several variables or those with operator arguments, of course if the above conjecture can be extended for these classes of generalized means.

Before giving an affirmative response that we are waiting for, we state some needed notions. A sequence $\left(m_{n}\right)_{n}$ of means will be called point-wise convergent (in short, $p$-convergent) if, for all $a, b>0$, the real sequence $\left(m_{n}(a, b)\right)_{n}$ converges. Setting $m_{\infty}(a, b)=$ $\lim _{n} m_{n}(a, b)$, it is easy to see that $m_{\infty}$ is a mean. Similarly, we define the point-wise monotonicity of $\left(m_{n}\right)_{n}$. By virtue of the double inequality

$$
\min (a, b) \leq m_{n}(a, b) \leq \max (a, b)
$$

we deduce that every $p$-increasing (resp. $p$-decreasing) sequence $\left(m_{n}\right)_{n}$ is $p$-convergent.

Now, let $m_{1}$ and $m_{2}$ be two given means and define the following two mean-sequences:

$$
\left\{\begin{array}{lll}
\Theta_{n+1}=\mathcal{R}\left(m_{1}, \Theta_{n}, m_{2}\right), & n \geq 0, & \Theta_{0}=m_{1} \\
\Upsilon_{n+1}=\mathcal{R}\left(m_{1}, \Upsilon_{n}, m_{2}\right), & n \geq 0, & \Upsilon_{0}=m_{2}
\end{array}\right.
$$

By mathematical induction, it is not hard to check that $\Theta_{n}$ and $\Upsilon_{n}$ are means for every $n \geq 0$. In the following, we study the $p$-convergence of the mean-sequences $\left(\Theta_{n}\right)_{n}$ and $\left(\Upsilon_{n}\right)_{n}$. We may state the next result.

Proposition 3.1 Let $m_{1}$ and $m_{2}$ be two stable means with $m_{1} \leq m_{2}$. Then the following mean-inequalities

$$
m_{1} \leq \cdots \leq \Theta_{n-1} \leq \Theta_{n} \leq \Upsilon_{n} \leq \Upsilon_{n-1} \leq \cdots \leq m_{2}
$$

hold for all $n \geq 1$. Consequently, the mean-sequences $\left(\Theta_{n}\right)_{n}$ and $\left(\Upsilon_{n}\right)_{n}$ both p-converge.

Proof Since $\Theta_{0}:=m_{1} \leq m_{2}:=\Upsilon_{0}$, we deduce by simple mathematical induction, with the help of (1.1), that $\Theta_{n} \leq \Upsilon_{n}$ for each $n \geq 0$. Now, using the fact that $m_{1}$ is stable, we can write, again with help of (1.1),

$$
\Theta_{0}=m_{1}=\mathcal{R}\left(m_{1}, m_{1}, m_{1}\right) \leq \mathcal{R}\left(m_{1}, m_{1}, m_{2}\right)=\mathcal{R}\left(m_{1}, \Theta_{0}, m_{2}\right)=\Theta_{1} .
$$

This, with mathematical induction, shows that $\Theta_{n-1} \leq \Theta_{n}$ for each $n \geq 1$. Analogously, we prove that $\Upsilon_{n} \leq \Upsilon_{n-1}$ for every $n \geq 1$. Summarizing, we deduce that $\left(\Theta_{n}\right)_{n}$ is a $p$-increasing sequence $p$-upper bounded by $m_{2}$, while $\left(\Upsilon_{n}\right)_{n}$ is a $p$-decreasing sequence $p$-lower bounded by $m_{1}$. Then the desired result follows, and so this completes the proof.

We explicitly notice that the above mean-sequences $\left(\Theta_{n}\right)_{n}$ and $\left(\Upsilon_{n}\right)_{n} p$-converge for all comparable means $m_{1}$ and $m_{2}$, i.e., $m_{1} \leq m_{2}$ (or $m_{2} \leq m_{1}$, see Remark 3.1 below). Now, a natural question arises from the above: under what conditions on $m_{1}$ and $m_{2}$ do the $p$-limits of $\left(\Theta_{n}\right)_{n}$ and $\left(\Upsilon_{n}\right)_{n}$ coincide? In what follows, we are interested in finding a positive answer to this question. 
Proposition 3.2 Let $m_{1}$ and $m_{2}$ be two means. Assume that $m_{1}$ is a cross mean, then the mean-sequences $\left(\Theta_{n}\right)_{n}$ and $\left(\Upsilon_{n}\right)_{n}$ satisfy the following relationship:

$$
\forall n \geq 0 \quad \Theta_{n+1}=\mathcal{M}\left(m_{1}, \Theta_{n}, \Upsilon_{n}\right):=m_{1}\left(\Theta_{n}, \Upsilon_{n}\right)
$$

Proof We use mathematical induction. For $n=0$, by (3.1) and Proposition 2.1, we have

$$
\Theta_{1}=\mathcal{R}\left(m_{1}, \Theta_{0}, m_{2}\right)=\mathcal{R}\left(m_{1}, m_{1}, m_{2}\right)=\mathcal{M}\left(m_{1}, m_{1}, m_{2}\right)=\mathcal{M}\left(m_{1}, \Theta_{0}, \Upsilon_{0}\right)
$$

Assume that (3.3) is true for $n$, by (3.1) we obtain

$$
\Theta_{n+2}=\mathcal{R}\left(m_{1}, \Theta_{n+1}, m_{2}\right)=\mathcal{R}\left(m_{1}, \mathcal{M}\left(m_{1}, \Theta_{n}, \Upsilon_{n}\right), m_{2}\right)
$$

This, with Theorem 2.2, yields

$$
\Theta_{n+2}=\mathcal{M}\left(m_{1}, \mathcal{R}\left(m_{1}, \Theta_{n}, m_{2}\right), \mathcal{R}\left(m_{1}, \Upsilon_{n}, m_{2}\right)\right)=\mathcal{M}\left(m_{1}, \Theta_{n+1}, \Upsilon_{n+1}\right)
$$

so proving the desired result.

Now, we are in a position to state the next result.

Theorem 3.3 Let $m_{1}$ and $m_{2}$ be two stable means with $m_{1} \leq m_{2}$. Assume further that $m_{1}$ is strict and a cross mean. Then, the mean-sequences $\left(\Theta_{n}\right)_{n}$ and $\left(\Upsilon_{n}\right)_{n}$ both $p$-converge to the same limit $m$ which is $\left(m_{1}, m_{2}\right)$-stabilizable and satisfying $m_{1} \leq m \leq m_{2}$.

Proof According to Proposition 3.1, the sequences $\left(\Theta_{n}\right)_{n}$ and $\left(\Upsilon_{n}\right)_{n}$ both $p$-converge. Call their limits $\Theta$ and $\Upsilon$, respectively. By the continuity of $m_{1}$, relationship (3.3) gives, when $n \rightarrow \infty, \Theta=m_{1}(\Theta, \Upsilon)$. This, with the fact that $m_{1}$ is strict, yields $\Theta=\Upsilon:=m$. Letting $n \rightarrow \infty$ in the first (or second) relation of (3.1), we obtain, with the help of continuity of $m_{1}, m=\mathcal{R}\left(m_{1}, m, m_{2}\right)$, which means that $m$ is $\left(m_{1}, m_{2}\right)$-stabilizable. Inequalities (3.2) imply that $m_{1} \leq m \leq m_{2}$, which completes the proof.

Corollary 3.4 Let $m_{1}$ and $m_{2}$ be as in the above theorem. Let $m$ be a $\left(m_{1}, m_{2}\right)$-stabilizable mean such that $m_{1} \leq m \leq m_{2}$. Then $m$ is the common p-limit of the above sequences $\left(\Theta_{n}\right)$ and $\left(\Upsilon_{n}\right)$.

Proof We show, by mathematical induction, that $\Theta_{n} \leq m \leq \Upsilon_{n}$ for all $n \geq 0$. For $n=0$, it is true by virtue of $\Theta_{0}=m_{1} \leq m \leq m_{2}=\Upsilon$. Assume that $\Theta_{n} \leq m \leq \Upsilon_{n}$, the recursive relations (3.1), with the help of (1.1), give

$$
\Theta_{n+1}=\mathcal{R}\left(m_{1}, \Theta_{n}, m_{2}\right) \leq \mathcal{R}\left(m_{1}, m, m_{2}\right) \leq \mathcal{R}\left(m_{1}, \Upsilon_{n}, m_{2}\right)=\Upsilon_{n+1}
$$

This, with the fact that $m$ is $\left(m_{1}, m_{2}\right)$-stabilizable, i.e., $m=\mathcal{R}\left(m_{1}, m, m_{2}\right)$, yields $\Theta_{n+1} \leq$ $m \leq \Upsilon_{n+1}$. It follows that $\Theta_{n} \leq m \leq \Upsilon_{n}$ for all $n \geq 0$. Since the sequences $\left(\Theta_{n}\right)$ and $\left(\Upsilon_{n}\right)$ both $p$-converge to the same limit, we deduce the desired result.

The above corollary tells us that every $\left(m_{1}, m_{2}\right)$-stabilizable mean is the common $p$-limit of the above sequences $\left(\Theta_{n}\right)$ and $\left(\Upsilon_{n}\right)$. This, with the uniqueness of the $p$-limit, implies immediately the next result, which gives an affirmative answer of the above conjecture. 
Corollary 3.5 Let $m_{1}$ and $m_{2}$ be as in the above theorem. Then there exists one and only one $\left(m_{1}, m_{2}\right)$-stabilizable mean $m$ such that $m_{1} \leq m \leq m_{2}$.

Remark 3.1 (i) If the means $m_{1}$ and $m_{2}$ are such that $m_{1} \geq m_{2}$, analogous results as those above can be stated in a similar way. We leave to the reader the task to formulate these results in a detailed manner. In particular, with convenient means $m_{1}$ and $m_{2}$, there exists one and only one $\left(m_{1}, m_{2}\right)$-stabilizable mean satisfying that $m_{2} \leq m \leq m_{1}$.

(ii) For $m_{1}$ and $m_{2}$ as in the above theorem, the last corollary tells us that the map $m \longmapsto$ $\mathcal{R}\left(m_{1}, m, m_{2}\right)$ has one and only one mean-fixed point.

Example 3.1 As already pointed, the mean $L$ is $(H, A)$-stabilizable. Following the above study, $L$ is the unique $(H, A)$-stabilizable mean satisfying $H \leq L \leq A$, and so $L$ can be characterized as the $p$-limit of an iterative algorithm involving the simple means $H$ and $A$. The same can be said for the other stabilizable means mentioned in Theorem 2.2. We leave it for the reader to give more details about this latter point in a similar manner as previously explained.

It is worth mentioning that the reader will do well in distinguishing between the following two situations:

(a) There exists one and only one $\left(m_{1}, m_{2}\right)$-stabilizable mean for suitable means $m_{1}$ and $m_{2}$ as previously showed.

(b) A given mean $m$ can be $\left(m_{1}, m_{2}\right)$-stabilizable and $\left(m_{1}^{\prime}, m_{2}^{\prime}\right)$-stabilizable for two distinct couples $\left(m_{1}, m_{2}\right)$ and $\left(m_{1}^{\prime}, m_{2}^{\prime}\right)$. Indeed, as already pointed before, the logarithmic mean $L$ is simultaneously $(A, G)$-stabilizable and $(H, A)$-stabilizable.

Finally, the following is of interest: Let $m_{1}$ and $m_{2}$ be as in the above theorem. For every mean $m$, we set

$$
\Lambda_{m_{1}}^{m_{2}}(m)=\mathcal{R}\left(m_{1}, m, m_{2}\right)
$$

Then, for fixed means $m_{1}$ and $m_{2}, \Lambda_{m_{1}}^{m_{2}}$ defines a map from the set of means into itself. If $\left(\Lambda_{m_{1}}^{m_{2}}\right)^{(n)}$ denotes the $n$-iterate of $\Lambda_{m_{1}}^{m_{2}}$, i.e., $\left(\Lambda_{m_{1}}^{m_{2}}\right)^{(0)}(m)=m$ and, for $n \geq 1$,

$$
\left(\Lambda_{m_{1}}^{m_{2}}\right)^{(n)}=\Lambda_{m_{1}}^{m_{2}} \circ \Lambda_{m_{1}}^{m_{2}} \circ \cdots \circ \Lambda_{m_{1}}^{m_{2}} \quad\left(n \text { times of } \Lambda_{m_{1}}^{m_{2}}\right)
$$

then the above study tells us that every $\left(m_{1}, m_{2}\right)$-stabilizable mean $m$ can be written as

$$
m=\lim _{n}\left(\Lambda_{m_{1}}^{m_{2}}\right)^{(n)}\left(m_{1}\right)=\lim _{n}\left(\Lambda_{m_{1}}^{m_{2}}\right)^{(n)}\left(m_{2}\right)
$$

for the point-wise limit. Further, the following iterative inequalities hold true (if $m_{1} \leq m_{2}$ ):

$$
\forall n \geq 1 \quad\left(\Lambda_{m_{1}}^{m_{2}}\right)^{(n-1)}\left(m_{1}\right) \leq\left(\Lambda_{m_{1}}^{m_{2}}\right)^{(n)}\left(m_{1}\right) \leq m \leq\left(\Lambda_{m_{1}}^{m_{2}}\right)^{(n)}\left(m_{2}\right) \leq\left(\Lambda_{m_{1}}^{m_{2}}\right)^{(n-1)}\left(m_{2}\right)
$$

Example 3.2 $L$ is $(H, A)$-stabilizable. Then we have

$$
\forall n \geq 1 \quad\left(\Lambda_{H}^{A}\right)^{(n-1)}(H) \leq\left(\Lambda_{H}^{A}\right)^{(n)}(H) \leq L \leq\left(\Lambda_{H}^{A}\right)^{(n)}(A) \leq\left(\Lambda_{H}^{A}\right)^{(n-1)}(A) .
$$


Simple computations lead to

$$
\Lambda_{H}^{A}(H)=\frac{2 G^{2}}{A+H}, \quad \Lambda_{H}^{A}(A)=\frac{3}{4} A+\frac{1}{4} H .
$$

We then obtain

$$
H \leq \frac{2 G^{2}}{A+H} \leq L \leq \frac{3}{4} A+\frac{1}{4} H \leq A,
$$

which refines $H \leq L \leq A$. The procedure can be continued for obtaining more iterative refinements for this latter double inequality.

\section{Competing interests}

The author declares that he has no competing interests.

\section{Acknowledgements}

Many thanks to the anonymous referee for bringing us some recent references. This work was supported by the Research Center of Taibah University (No. 4625/2013).

Received: 24 May 2013 Accepted: 5 September 2013 Published: 07 Nov 2013

\section{References}

1. Raïssouli, M: Stability and stabilizability for means. Appl. Math. E-Notes 11, 159-174 (2011)

2. Raïssouli, M: Approaching the power logarithmic and difference means by algorithms involving the power binomial mean. Int. J. Math. Math. Sci. 2011, Article ID 687825 (2011)

3. Raïssouli, M: Stabilizability of the Stolarsky mean and its approximation in terms of the power binomial mean. Int. J. Math. Anal. 6(18), 871-881 (2012)

4. Raïssouli, M: Refinements for mean-inequalities via the stabilizability concept. J. Inequal. Appl. 2012, 55 (2012)

5. Bullen, PS: Handbook of Means and Their Inequalities. Kluwer Academic, Dordrecht (2003)

6. Chu, Y-M, Long, B-Y: Bounds for the Neuman-Sándor mean using power and identric means. Abstr. Appl. Anal. 2013, Article ID 832591 (2013)

7. Chu, Y-M, Long, B-Y, Gong, W-M, Song, Y-Q: Sharp bounds for Seiffert and Neuman-Sándor means in terms of generalized logarithmic means. J. Inequal. Appl. 2013, 10 (2013)

8. Chu, Y-M, Shi, M-Y, Jiang, Y-P: Optimal inequalities for power, harmonic and logarithmic means. Bull. Iran. Math. Soc. 38(3), 597-606 (2012)

9. Chu, Y-M, Wang, M-K: Optimal Lehmer mean bounds for the Toader mean. Results Math. 61(3-4), $223-229$ (2012)

10. Chu, Y-M, Wang, M-K, Wang, Z-K: Best possible inequalities among harmonic, geometric, logarithmic and Seiffert means. Math. Inequal. Appl. 15(2), 415-422 (2012)

11. Chu, Y-M, Wang, M-K, Qiu, S-L: Optimal combinations bounds of root-square and arithmetic means for Toader mean. Proc. Indian Acad. Sci. Math. Sci. 122(1), 41-51 (2012)

12. Chu, Y-M, Xia, W-F: Two optimal double inequalities between power mean and logarithmic mean. Comput. Math. Appl. 60(1), 83-89 (2010)

13. Chu, Y-M, Wang, M-K, Gong, W-M: Two sharp double inequalities for Seiffert mean. J. Inequal. Appl. 2011, 44 (2011)

14. Hu, H-N, Tu, G-Y, Chu, Y-M: Optimal bounds for Seiffert mean in terms of one-parameter means. J. Appl. Math. 2012, Article ID 917120 (2012)

15. Li, Y-M, Long, B-Y, Chu, Y-M: Sharp bounds for the Neuman-Sándor mean in terms of generalized logarithmic mean. J. Math. Inequal. 6(4), 567-577 (2012)

16. Neuman, E: On one-parameter family of bivariate means. Aequ. Math. 83(1-2), 191-197 (2012)

17. Neuman, E: A note on a certain bivariate mean. J. Math. Inequal. 6(4), 637-643 (2012)

18. Neuman, E: Inequalities for the Schwab-Borchardt mean and their applications. J. Math. Inequal. 5(4), 601-609 (2011)

19. Neuman, E, Sándor, J: Companion inequalities for certain bivariate means. Appl. Anal. Discrete Math. 3(1), 46-51 (2009)

20. Qiu, Y-F, Wang, M-K, Chu, Y-M: Two sharp inequalities for Lehmer mean, identric mean and logarithmic mean. J. Math. Inequal. 5(3), 301-306 (2011)

21. Raïssouli, M, Sándor, J: On a method of construction of new means with applications. J. Inequal. Appl. 2013, 89 (2013)

22. Shi, H-X, Long, B-Y, Chu, Y-M: Optimal generalized Heronian mean bounds for the logarithmic mean. J. Inequal. Appl. 2012, 63 (2012)

23. Wang, M-K, Wang, Z-K, Chu, Y-M: An optimal double inequality between geometric and identric means. Appl. Math. Lett. 25(3), 471-475 (2012)

24. Zhao, T-H, Chu, Y-M, Liu, B-Y: Optimal bounds for Neuman-Sándor mean in terms of the convex combinations of harmonic, geometric, quadratic, and contraharmonic means. Abstr. Appl. Anal. 2012, Article ID 302635 (2012) 